\section{IMMUNE INFILTRATES ARE ASSOCIATED WITH CLINICAL OUTCOMES IN PATIENTS WITH RESECTABLE SOFT TISSUE SARCOMA (STS) TREATED WITH NEOADJUVANT IMMUNE CHECKPOINT BLOCKADE (ICB)}

Emily Keung*, Elise Nassif, Heather Lin, Alexander Lazar, Wei-Lien Wang, Edwin Parra, Cibelle Lima, Ignacio Wistuba, Ashleigh Guadagnolo, Andrew Bishop, Valerae Lewis, Keila Torres, Kelly Hunt, Barry Feig, Christopher Scally, Ahmed Al Rawi, Shadarra Crosby, Grace Mathew, Davis Ingram, Khalida Wani, Jennifer Wargo, Neeta Somaiah, Christina Roland. The University of Texas MD Anderson Cancer Center, Houston, TX, USA

Background Recurrences are common after surgery for localized STS. ${ }^{1}{ }^{2}$ ICB has shown activity in metastatic undifferentiated pleomorphic sarcoma (UPS) and dedifferentiated liposarcoma (DDLPS $)^{3}$ with intratumoral B-cells associated with improved outcomes. ${ }^{4}$ We assessed biomarkers of response in a novel phase II trial of neoadjuvant ICB for resectable UPS and DDLPS.

Methods DDLPS $(n=17)$ and UPS $(n=10)$ patients were randomized to neoadjuvant nivolumab or ipilimumab+nivoluumab, with UPS patients receiving concurrent radiotherapy ${ }^{5}$ (figure 1). Baseline and on-treatment tumor biopsies were obtained; primary endpoint was pathologic response defined as $>30 \%$ hyalinization at surgery after optimal cutoff determination. ${ }^{6}$ We examined association of tumor-infiltrating immune cells, assessed by immunohistochemistry and multiplex immunofluorescence (mIF), with pathologic response, survival and resistance as defined by Society for Immunotherapy of Cancer Criteria. ${ }^{7}$ Statistical analysis included Kruskal-Wallis, Wilcoxon and McNemar tests. Log-rank tests were performed to compare relapse-free survival (RFS) and overall survival (OS).

Results Pathologic response was seen in 18\% DDLPS $(\mathrm{N}=3$ / 17) and 90\% UPS $(\mathrm{N}=9 / 10)$ patients (figure 2). At a median follow-up of 23 months from treatment initiation, 12 (44\%) patients (9 DDLPS, 3 UPS) relapsed and 4 (14\%) died due to recurrence (3 DDLPS, 1 UPS). The percentage of DDLPS tumors with CD20+/CD21+ B-cell infiltration increased with ICB (baseline: 19\%, surgery: 50\%; $p=0.056$; figure 3 ) and presence of B-cells at surgery for DDLPS displayed a trend toward longer median RFS (Not Reached [NR], 95\% CI 15NR months versus 13.4 months, 95\% CI 3.5-NR; $p=0.13$ ). All DDLPS patients with B-cells at surgery are alive whereas median OS in absence of B-cells was 28 months $(p=0.045)$. Two UPS patients had baseline intratumoral B-cells but none were found at surgery, presumably because B-cells are radiosensitive; neither have relapsed (follow-up: 33 and 31 months). By mIF (figure 4), tumors with baseline infiltration of $\mathrm{CD} 3+\mathrm{CD} 8+/ \mathrm{CD} 3+>17 \%$ had longer RFS $(\mathrm{p}=0.0038$; figure 5). Pathologic non-responders had higher density of baseline CD3+FoxP3+CD8- and on-treatment CD3+CD45RO + FoxP3 +CD8- lymphocytes $(\mathrm{p}=0.037$ and $\mathrm{p}=0.012$, respectively; figure 6). Furthermore, primary resistant STS had higher baseline CD3 +FoxP3+CD8- cell density $(p=0.068)$; STS with secondary resistance had higher density of CD3 + FoxP3 +CD8- cell density at surgery $(p=0.036$; figure 6$)$.

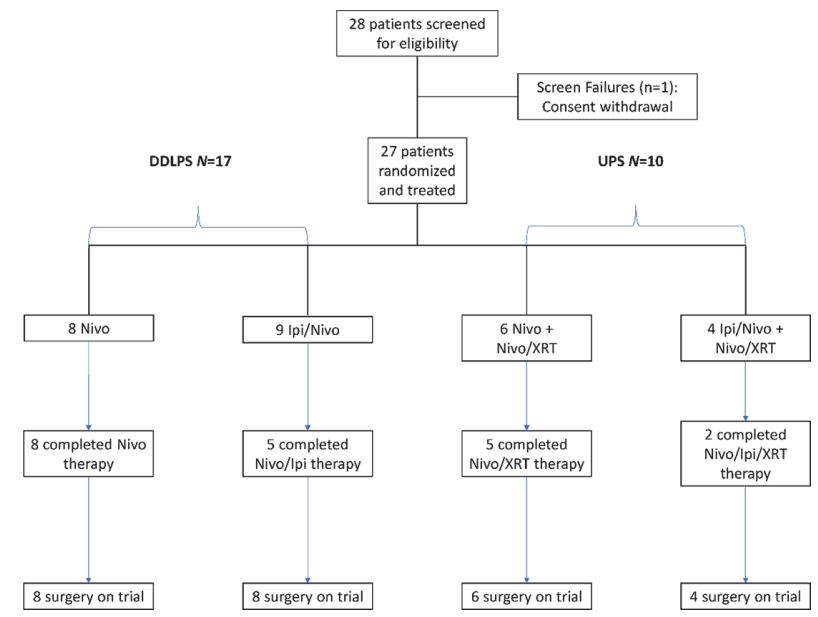

Abstract 379 Figure 1 Trial schema. Patients with DDLPS were randomized to receive either three cycles of nivolumab monotherapy (3mg/kg every 2 weeks) or one cycle of combination nivolumab/ ipilimumab (nivolumab $3 \mathrm{mg} / \mathrm{kg}$ and ipilimumab $1 \mathrm{mg} / \mathrm{kg}$ ) followed by two cycles of nivolumab monotherapy (3mg/kg every 2 weeks). Patients with UPS were randomized to receive either one cycle of nivolumab followed by concurrent radiation therapy (50Gy) with three cycles of nivolumab (3mg/kg every 2 weeks) or one cycle of combination nivolumab/ipilimumab (Nivolumab $3 \mathrm{mg} / \mathrm{kg}$ and Ipilimumab $1 \mathrm{mg} / \mathrm{kg}$ ) followed by concurrent radiation therapy (50Gy) with three cycles of Nivolumab (3mg/kg every 2 weeks).

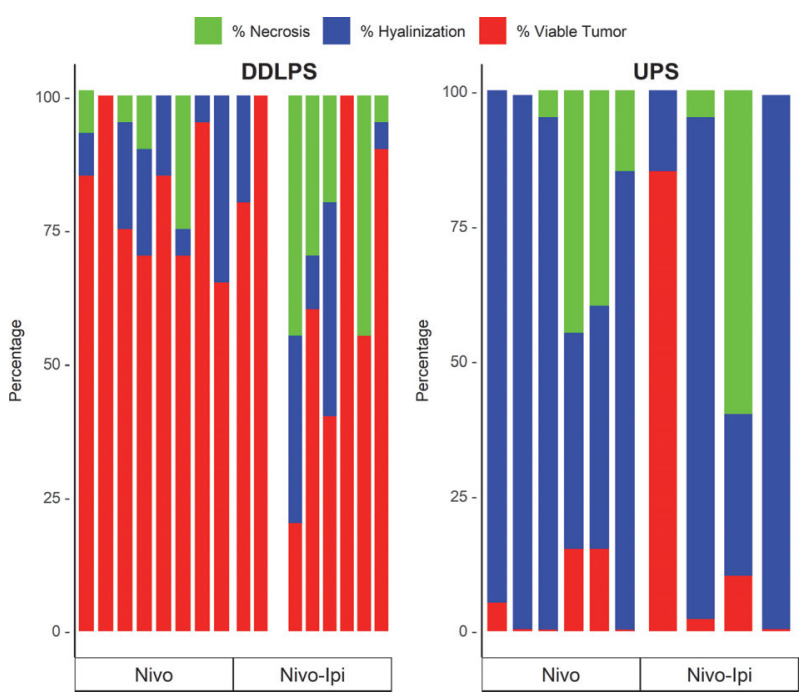

Abstract 379 Figure 2 Pathologic response to preoperative immune checkpoint blockade by treatment arm. The primary endpoint of this study was pathologic response as measured by percent tumor hyalinization. In the intention-to-treat population of all 27 randomized patients, median percent hyalinization was $8 \%(95 \% \mathrm{Cl} 0 \%-20 \%)$ in the DDLPS cohort and $89 \%(95 \% \mathrm{Cl} 30 \%-99 \%)$ in the UPS cohort. There were no differences in percent hyalinization between nivolumab monotherapy and combination nivolumab/ipilimumab treatment arms in either cohort. Median residual viable tumor in DDLPS was $77.5 \%(95 \%$ $\mathrm{Cl} 60 \%-95 \%)$ and $3.5 \%(95 \% \mathrm{Cl} 0-15 \%)$ in UPS cohorts and was similar between nivolumab monotherapy and combination ipilimumab/ nivolumab treatment arms. 
A

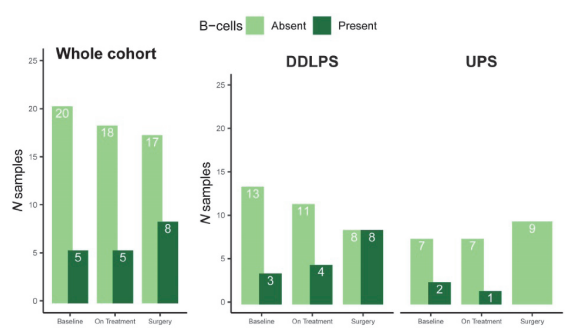

B
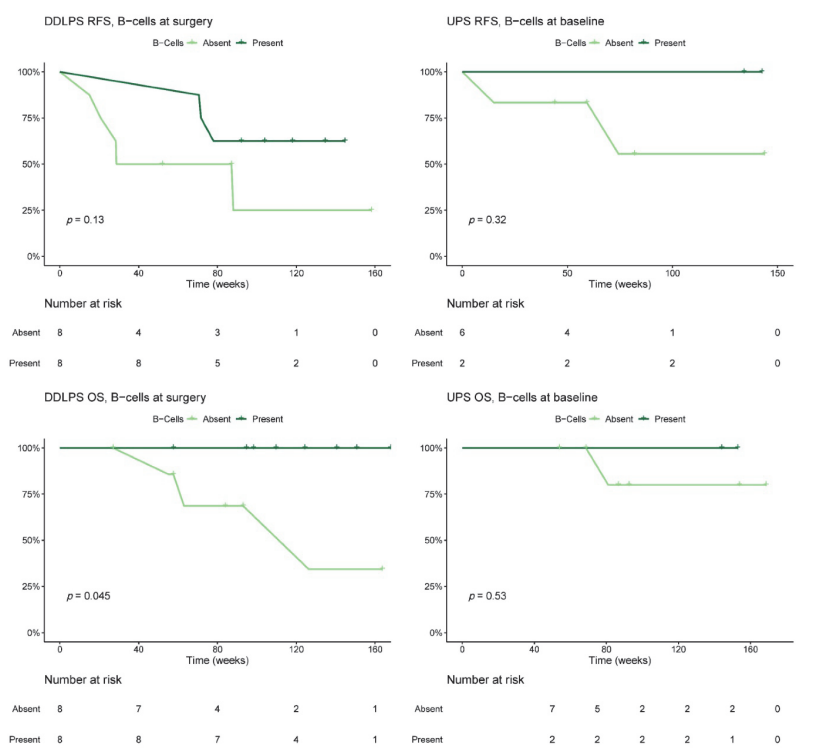

hesent

Abstract 379 Figure 3 Intratumoral B-cells kinetics and association with survival. (A) Number of samples positive and negative for B-cell staining at each clinical time point in the whole cohort (left panel) and histotype-specific (right panel) as assessed and verified by CD20 and CD21 immunohistochemistry, respectively. Increase in the number of Bcell positive samples in DDLPS with immune checkpoint blockers. Decrease in the UPS group due to radiosensitivity. (B) Association between presence of B-cells and relapse-free survival (RFS, top panels) and overall survival (OS, bottom panels). DDLPS patients with intratumoral B-cells at surgery experienced longer RFS and OS. UPS patients with intratumoral B-cells at baseline experienced longer RFS and $O S$.

${ }^{*} p$-values are Log-rank
A

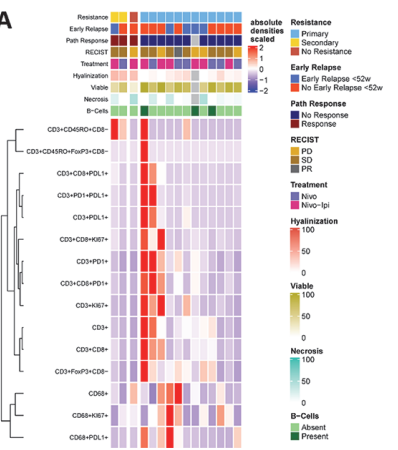

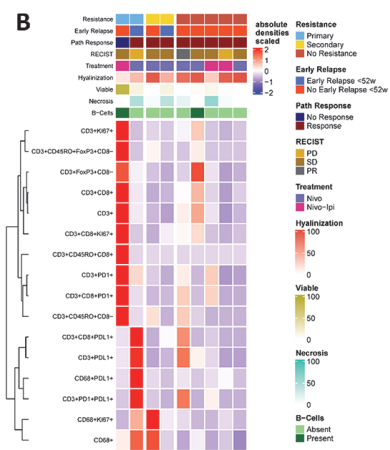

Abstract 379 Figure 4 Association of baseline tumor immune infiltrate with clinical outcomes. Baseline tumor infiltrating immune cells were assessed and quantified by multiplexed immunofluorescence. Densities have been scaled and patients were classified by resistance according to Society for Immunotherapy of Cancer (SITC) criteria. Immune cells are grouped by hierarchical clustering correlation using Spearman's coefficient. (A) Heatmap of absolute densities of immune infiltrating cells at baseline in DDLPS. Three patients had pathologic response, and only one of these patients did not relapse and was considered non-resistant. (B) Heatmap of absolute densities of immune infiltrating cells at baseline in UPS. One patient did not have pathologic response, two patients displayed early relapse and one patient had relapse after one year.
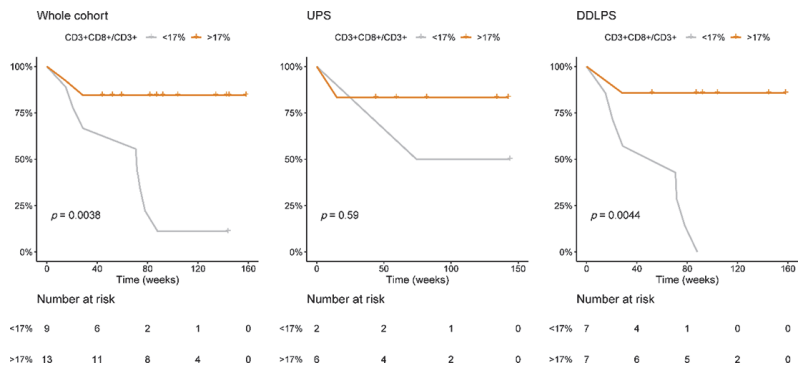

Abstract 379 Figure 5 Cytotoxic lymphocytes at baseline are associated with longer relapse-free survival. Kaplan Meier curves of relapse-free survival according to relative density of $\mathrm{CD} 3+\mathrm{CD} 8+/ \mathrm{CD} 3+>$ $17 \%$ at baseline. ${ }^{*}$ p-values are Log-rank.

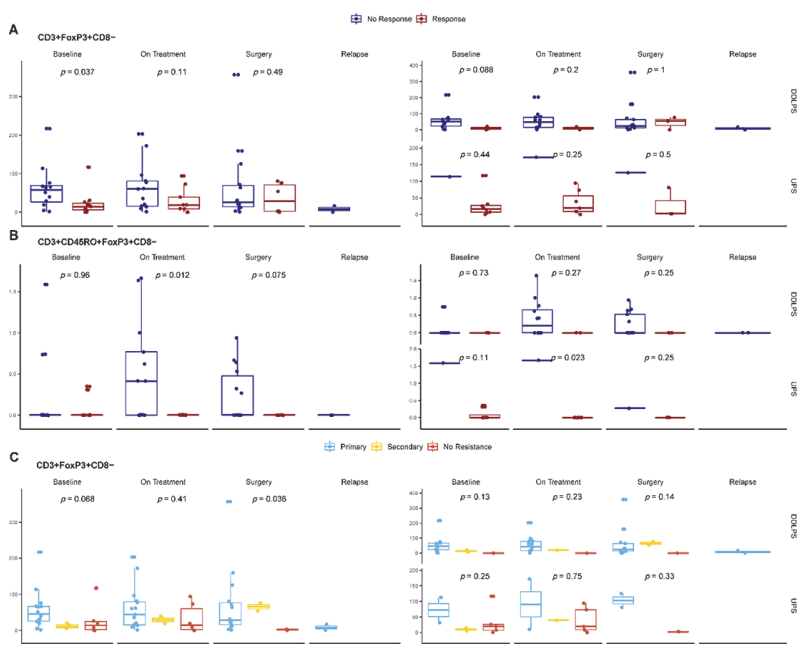

Abstract 379 Figure 6 T-regulatory cells are associated with poorer pathologic response and resistance to immune checkpoint blockade. (A) Absolute densities of regulatory T cells CD3+FoxP3+CD8- lymphocytes by pathological response at each clinical time point (entire cohort, left panel; by histotype, right panel). * $p$-values are Wilcoxon. (B) Absolute densities of memory/regulatory T-cells CD3+CD45R0+FoxP3+CD8lymphocytes by pathological response at each clinical time point (entire cohort, left panel; by histotype, right panel). * ${ }^{*}$-values are Wilcoxon. (C) Absolute densities of regulatory T cells CD3+FoxP3+CD8- lymphocytes by resistance as defined by SITC criteria at each clinical time point (entire cohort, left panel; by histotype, right panel). " $p$-values are Kruskal Wallis.

Conclusions B-cells at baseline in UPS and at surgery in DDLPS and cytotoxic $\mathrm{T}$ cells $\mathrm{CD} 3+\mathrm{CD} 8+$ at baseline are associated with better survival outcomes. T-regulatory cells are associated with poorer pathologic response and resistance to neoadjuvant ICB for DDLPS and UPS.

Acknowledgements This study was supported by Bristol Myers Squibb. EZK received support from the QuadW Foundation. EFN received support from Fondation pour la Recherche 


\section{Abstracts}

Medicale and Fondation Nuovo-Soldati. CLR received support from American College of Surgeons.

Trial Registration clinicaltrials.gov unique identifier: NCT03307616

\section{REFERENCES}

1.. Blay JY, Honore C, Stoeckle $E$, et al. Surgery in reference centers improves survival of sarcoma patients: a nationwide study. Ann Oncol 2019;30:1407.

2. . Gronchi A, Palmerini E, Quagliuolo V, et al. Neoadjuvant chemotherapy in highrisk soft tissue sarcomas: final results of a randomized trial from Italian (ISG), Spanish (GEIS), French (FSG), and Polish (PSG) sarcoma groups. Journal of Clinical Oncology 2020;38:2178-2186.

3.. Tawbi HA, Burgess $M$, Bolejack $V$, et al. Pembrolizumab in advanced soft-tissue sarcoma and bone sarcoma (SARC028): a multicentre, two-cohort, single-arm, open-label, phase 2 trial. The Lancet Oncology 2017;18:1493-1501.

4.. Petitprez F, de Reynies A, Keung EZ, et al. B cells are associated with survival and immunotherapy response in sarcoma. Nature 2020;577:556-560.

5.. Keung EZ, Lazar AJ, Torres KE, et al. Phase II study of neoadjuvant checkpoint blockade in patients with surgically resectable undifferentiated pleomorphic sarcoma and dedifferentiated liposarcoma. BMC Cancer 2018;18.

6.. Schaefer I-M, Hornick JL, Barysauskas CM, et al. Histologic appearance after preoperative radiation therapy for soft tissue sarcoma: assessment of the European organization for research and treatment of cancer-soft tissue and bone sarcoma group response score. International Journal of Radiation Oncology ${ }^{*}$ Biology ${ }^{*}$ Physics 2017;98:375-383.

7. . Kluger HM, Tawbi HA, Ascierto ML, et al. Defining tumor resistance to PD-1 pathway blockade: recommendations from the first meeting of the SITC immunotherapy resistance taskforce. J Immunother Cancer 2020;8.

Ethics Approval This study was approved by MD Anderson Cancer Center Institutional Review Board; approval number 2017-0143.

http://dx.doi.org/10.1136/jitc-2021-SITC2021.379 